\title{
The IRB Challenge for Practice-based Research: Strategies of the American Academy of Family Physicians National Research Network (AAFP NRN)
}

\author{
Deborah G. Graham, MSPH, Mindy S. Spano, and Brian Manning, MPH, CHES
}

Investigators for multisite research studies conducted in practice-based research networks face numerous challenges associated with Institutional Review Boards (IRBs) and human subjects protection. The American Academy of Family Physicians National Research Network (AAFP NRN) has adopted strategies to deal with some of these challenges, including creating an open, honest relationship with the new American Academy of Family Physicians Institutional Review Board (AAFP IRB); creating procedures for members who are not required to report to a local IRB; handling most of the IRB application submission and tracking tasks for our members who must submit applications to local IRBs; and working with the AAFP IRB to make required human subjects training relevant to our practices. However, these are only temporary solutions. It is time to begin working toward a permanent solution. As such, the AAFP, the AAFP NRN, and the AAFP IRB have begun discussing the possibility of adopting an alternative model of central IRB review, which would facilitate practice-based research in family medicine and which would encourage rather than discourage family medicine practices to participate in research studies that will further the discipline. (J Am Board Fam Med 2007;20:181-187.)

\section{Introduction and Background}

The American Academy of Family Physicians National Research Network (AAFP NRN), a national practice-based research network (PBRN) for family medicine, collaborates with research investigators, practices, and individuals to study questions relevant to both family medicine practices and community-based family medicine patients. AAFP NRN membership includes approximately 350 clinicians and study coordinators from 180 practices in 50 states and Canadian provinces. ${ }^{1}$ Some of the benefits of this arrangement include enhanced generalizability of results, pooling of resources, rapid patient recruitment, and collaborative opportunities. Although there are many benefits, there are common challenges and limitations encountered as

This article was externally peer-reviewed.

Submitted 29 June 2006; revised 27 October 2006; accepted 9 November 2006.

From the American Academy of Family Physicians $\mathrm{Na}$ tional Research Network, Leawood, KS.

Conflict of interest: none declared.

Previous presentation: This article is based on a presentation made at the American Academy of Family Physicians National Research Network 2006 Convocation of Practices and Networks, Dallas, TX, February 23-26, 2006.

Corresponding author: Deborah G. Graham, MSPH, 11400 Tomahawk Creek Parkway, Leawood, KS 66211 (Email: dgraham@aafp.org). a result of conducting multisite research projects at the national level. Frequent challenges arise around coordinating activities and incorporating changes across sites. One ubiquitous challenge across studies/projects is managing and coordinating issues related to institutional review board (IRB) reviews and the protection of human subjects.

There are a myriad of challenges associated with IRB and human subjects issues that project teams must resolve when conducting multisite studies. Most health systems, academic institutions, and hospitals have their own IRB. Most of these IRBs, as well as the AAFP IRB, require that they review and approve each study in which a clinician in their system participates. Consequently, for a multisite study in which 30 practices participate, generally at least 15 to 20 distinct IRBs require an individual application and review.

IRBs vary significantly in submission requirements and procedures, application formats, application questions, and review procedures for IRB applications. $^{2-8}$ Green, Lowery, Kowalski, and Wyszewainski ${ }^{5}$ observed 4 categories of issues impacting a multisite observational study: (1) recruitment, retention, and communication issues with local site principal investigators, a position required by local IRBs which is not meaningful to PBRN 
studies and which adds unnecessary burden to these participating physicians; (2) wide variation in standards applied to review and approval of IRB applications; (3) multiple returns for revision of applications, consent documents, and ancillary forms, including varying minor changes to individual consent forms according to language required by different IRBs; and (4) process failures (long turnaround times, lost paperwork, difficulty in obtaining necessary forms, nonavailability of key personnel at IRBs). These issues impose significant burdens on administrative time and resources at both the practice level and the PBRN staff level, cause delays in rolling out studies, and prevent some practices from even participating in PBRN research studies.

In addition to these IRB challenges, PBRNs face other broad challenges at various levels of the network structure (eg, clinician, network, and institutional). These challenges often affect and may interact with IRB and human subject issues. Examples of PBRN challenges impacted by IRB procedures include (1) involving busy clinicians in research projects, (2) not disrupting practice flow with projects, and (3) managing limited resources (budget, time, and personnel). ${ }^{9-11}$

Many researchers have suggested that a more effective way to address these difficulties is to adopt a centralized IRB review model. ${ }^{3-5,7,8}$ In November 2005, the National Institutes of Health (NIH), the Office of Human Research Protections (OHRP), and other key groups convened a workshop on Alternative Models of IRB Review to address this issue. ${ }^{12}$ Consequently, the AAFP and the AAFP IRB have held preliminary discussions to explore the issue of alternative IRB models for family medicine. In this paper we address 2 issues: (1) strategies that the AAFP NRN currently implements to address IRB and human subjects issues in a PBRN context; and (2) future directions that the AAFP is investigating to address the adoption of an alternative centralized IRB review model.

\section{AAFP NRN Strategies AAFP IRB}

The AAFP IRB was activated on January 2, 2006. The AAFP IRB is a diverse body which includes 7 members, 4 of whom are internal to the AAFP (2 physicians, 1 educator, and 1 researcher) and 3 external members from the Kansas City area com-
Table 1. Affiliation Status of AAFP NRN Members

\begin{tabular}{lcc}
\hline & $\begin{array}{c}\text { Not Required } \\
\text { to Report to } \\
\text { Local IRB }\end{array}$ & $\begin{array}{c}\text { Required to } \\
\text { Report to } \\
\text { Local IRB }\end{array}$ \\
\hline All AAFP NRN members & $52 \%$ & $48 \%$ \\
Active AAFP NRN members & $40 \%$ & $60 \%$ \\
\hline
\end{tabular}

munity, including an ethicist, the Vice Provost for Research at a local university, and an administrator of a local academic IRB. Because most of the research conducted within the AAFP is conducted by the AAFP NRN, the Network played an active role in planning for and developing the AAFP IRB. Consequently, the IRB is extremely sensitive to PBRN issues, and its members took it on themselves to become informed about PBRN research. This relationship has allowed us to develop procedures that ensure compliance with the federal policy for the protection of human subjects ${ }^{13}$ while limiting the burden on participating practices. This relationship has also created a 2-way understanding of research methodology, such that the AAFP NRN does not have to continuously explain methodology to unfamiliar IRB members and, likewise, the IRB has helped the AAFP NRN develop an acceptable and recognized set of standard protocols for protecting human subjects.

When the AAFP IRB was developed it also was created to serve as a HIPAA Privacy Board. ${ }^{14}$ This allows the AAFP NRN to submit their protocols to only one board for both research and privacy issues. To date there have been no problems with other institutions accepting authorization forms or waivers of authorization which have been approved by the AAFP IRB.

\section{Working with Practices That Do Not Report to a Local IRB}

When clinicians first join the AAFP NRN, we ask whether they are required by their institution to report to a local IRB. Table 1 shows that just over one-half of the members indicate they are not required to report to a local IRB, although only $40 \%$ of our active members fit into this category.

If clinicians do not report to a local IRB, we ask them to sign an Unaffiliated Investigator Agreement (UIA) with the AAFP NRN. The UIA was developed in cooperation with the AAFP IRB and states that the clinician will (1) review and complete 


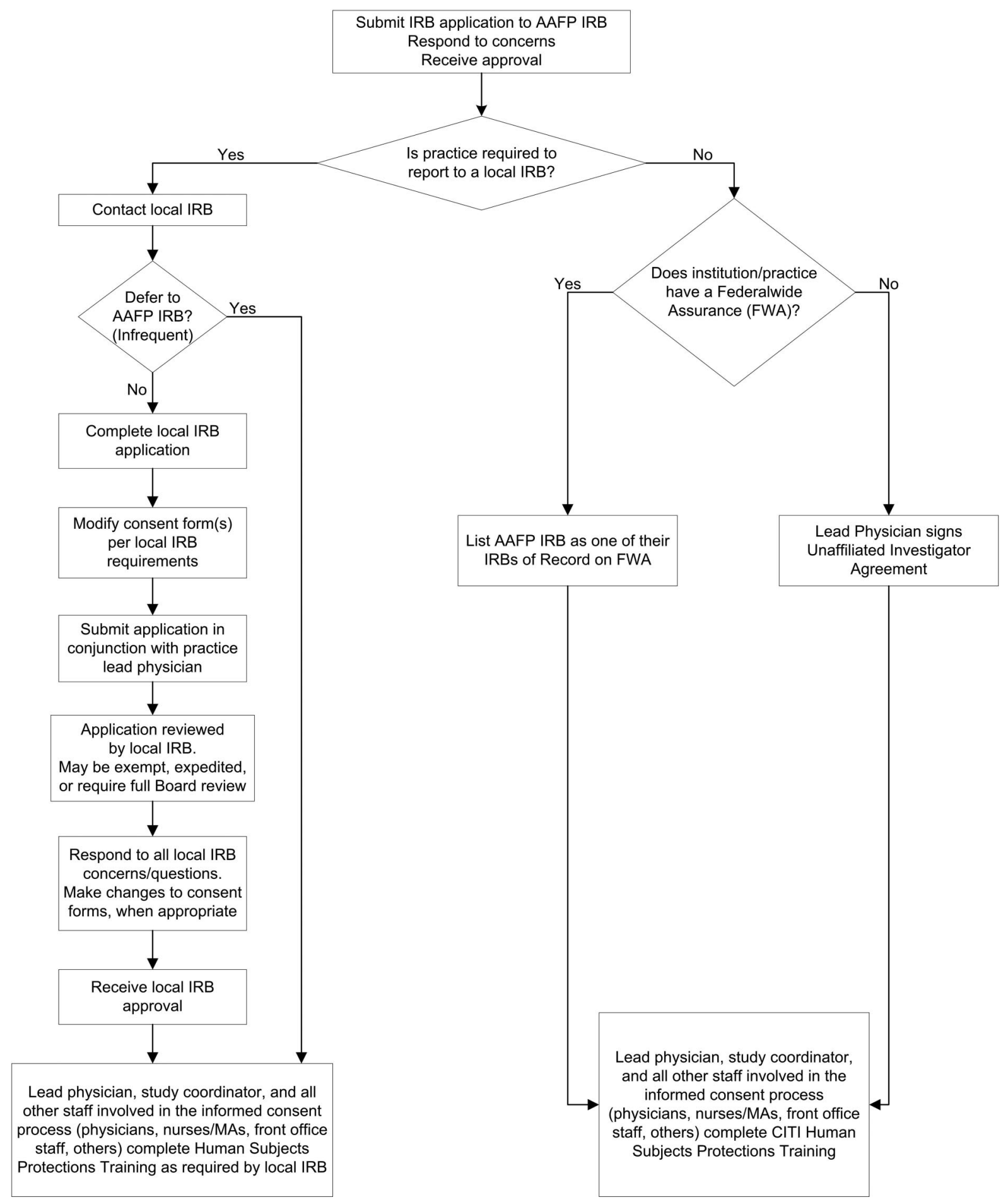

Figure 1. Flow of Institutional Review Board (IRB) work for the American Academy of Family Physicians National Research Network (AAFP NRN).

the appropriate human subjects protection training before participating in an AAFP NRN research study; (2) comply with the procedural standards of the US Department of Health and Human Services regulations for the protection of human subjects ${ }^{13}$; and (3) abide by all determinations of the IRB. Once the agreement is signed by all required parties, the AAFP IRB becomes the IRB of record for those AAFP NRN members and no additional IRB applications or HIPAA forms need to be submitted. 
The flow of IRB work for unaffiliated investigators is described in Figure 1. The clinicians sign the UIA upon enrollment in the AAFP NRN, and the agreement is good for a 3 -year period. The AAFP NRN staff tracks all signed UIAs in a database and contacts the members 60 to 90 days before expiration. Members are required to renew their UIA before participating in research studies.

From the IRB's point of view, it is important for them to be familiar with the practices that participate in NRN studies. For each research study, the AAFP NRN is required to notify the AAFP IRB of the name and location of all participating practices. This, coupled with the IRB's familiarity with practice-based research, contributes to the IRB's "knowledge of local context," that is, their knowledge of the environment and context in which each study will be implemented. Because of its newness, the IRB has not yet initiated audit procedures of protocols that it has approved to ensure compliance. These procedures have been developed and will be part of the ongoing review process.

\section{Human Subjects Protection}

When AAFP NRN unaffiliated members sign the UIA, they agree to complete human subjects training before participating in an AAFP NRN study. The OHRP requires that all key personnel of a research study complete human subjects training. OHRP defines "key personnel" as "all individuals responsible for the design and conduct of the study." 15 The AAFP IRB interpreted this statement to include the practice lead physicians and study coordinators for each study, as well as practice staff who obtain consent from patients to participate in a study. The AAFP IRB selected the Web-based CITI program ${ }^{16}$ as the human subjects training program that all key personnel must complete. Although the CITI program is widely used and informative in terms of human subjects research and federal regulations, it is not always accessible to busy clinicians and practice staff, and it can require several hours to complete all modules. The AAFP NRN recognized this and worked with the AAFP IRB to select the appropriate level of training for clinicians and practice staff that is most relevant to PBRN research. The AAFP IRB requires that the key practice personnel of all unaffiliated practices complete the following 4 modules for all studies: (1) History and Ethical Principles; (2) Informed Consent; (3) Records-Based Research; and (4)
HIPAA and Human Subjects Research. In addition, additional modules may be required, depending on unique characteristics of a particular protocol.

The AAFP NRN took steps to make the required human subjects training more accessible for busy clinicians and staff by offering alternative training formats. In addition to the Web-based training, we can provide a paper version of the modules. We also make available an electronic slideshow of human subjects training that the lead physician and study coordinator can present to the practice. We generally ask that all clinicians participating in a study and the staff who are involved in the patient consenting process complete the training. Many of the training formats originated from suggestions by clinicians and practice staff regarding efficient use of their time. We try to make this process as straightforward and trouble-free for the participating practices as possible. Once clinicians and staff complete the training, we keep a copy of their certificates of training completion. We also $\log$ and track the training completion date in a database and notify members and staff 60 to 90 days before expiration. The AAFP IRB requires that human subjects training certificates be renewed every 3 years, which benefits the clinicians by permitting them to renew the training at the same time they renew their UIAs.

\section{Working with Practices That Are Required to Report to a Local IRB}

When selecting sites for a study we generally choose practices with varied backgrounds. There are always some practices that are part of a larger health system, university system, or hospital system and that must report to a local IRB (see Table 1). In these instances, the AAFP NRN uses a centralized process for submitting and tracking the various local IRB applications. To reduce confusion, all submissions to local IRBs are coordinated through the project staff of the given study. When working with local IRBs, the project staff is responsible for completing all IRB materials for the physician. When the project staff receives approval from the AAFP IRB, he or she begins the process of contacting all practices in the study that are affiliated with a local IRB to begin the submission process. A copy of the AAFP IRB approval letter is always included in the submission to the local IRBs.

We submit applications and materials directly to the local IRBs when permitted, rather than go 


\begin{tabular}{lll}
\hline & Primary IRB* & \multicolumn{1}{c}{ Local IRBs } \\
\hline $\begin{array}{l}\text { Estimated AAFP NRN staff time to complete } \\
\text { initial IRB applications }\end{array}$ & 8 to 14 hours & 3 to 10 hours \\
Typical type of review & Expedited & Expedited \\
$\begin{array}{l}\text { Approximate process time from submission to } \\
\text { approval for initial IRB application }\end{array}$ & 44 days & 25 days for each application \\
\hline
\end{tabular}

* Currently the AAFP IRB. Prior to January 2006, the University of Missouri-Kansas City's Social Sciences IRB and Adult Health Sciences IRB reviewed protocols for the AAFP NRN.

through the practice clinicians. A direct line of communication between the AAFP NRN staff and the local IRBs generally allows for a more efficient submission and approval process. However, some local IRBs will only communicate with the practice clinician, not with the AAFP NRN staff. In these latter situations, we assist the practice with the completion and submission process as much as possible. The AAFP NRN project staff keep copies of the IRB applications, approval letters, and correspondence from the local IRBs for tracking purposes. We also request a letter from the practices that certifies that all key personnel have completed the human subjects training required by the local IRB, describes the type of training, and affirms that all their certificates are on file and can be viewed on request by the AAFP NRN or AAFP IRB. Table 2 describes the length of time generally required to secure initial approval for AAFP NRN studies.

The project staff are also responsible for tracking, completing, and if possible, submitting annual project renewal applications to the local IRBs for a given study. They track all approval/renewal dates throughout the project. If they are not contacted by the local IRB or affiliated practice at least 2 months from expiration, the project team follows up with the appropriate practice lead physician or study coordinator on the status of the renewal. After clarifying and coordinating any changes to the protocol, the project staff typically complete the renewal application for the affiliated practice. As with the initial application, the submission process often varies. In some instances, the local IRB allows the AAFP NRN to directly submit a renewal application; in other instances, the lead physician may wish to participate in the submission process; and in some situations, the local IRB refuses all contact with the AAFP NRN and communicates only with the affiliated practice staff. Whenever possible, the project staff work to close the protocol after data collection has ended. The AAFP IRB allows a protocol to be closed and analyses to continue as long as the patient information has been de-identified. However, we have encountered cases in which a local IRB will not close a project until all analyses are completed.

\section{Future Solutions}

The AAFP NRN has developed procedures to minimize the IRB burden for its members while at the same time respecting human subjects protections issues and regulatory compliance. These procedures have been described above. However, there are several major weaknesses to this model, including but not limited to a significant drain on PBRN resources. Some of these weaknesses have been discussed in this article; many have been discussed previously. ${ }^{3-8}$ To address many of these weaknesses, the AAFP NRN and the authors cited above recommend that a system of centralized IRB review be investigated as a possible alternative IRB review model.

Practice-based research is important to the AAFP and is included as one of the "basket of services" in the New Model of Family Medicine. ${ }^{17}$ As such, the AAFP, the AAFP NRN, and the AAFP IRB have had preliminary discussions concerning instituting an alternative model of IRB oversight that would facilitate practice-based research in family medicine and encourage rather than discourage family medicine clinicians to participate in research studies to further the discipline. Specifically, we are discussing models that include a central review component that were presented at the Alternative Models of IRB Review Workshop in November $2005 .^{12}$ These include the following models:

- An institution relies on the review by another institution's IRB for a particular study; 
- a local IRB participates in a facilitated review for a multisite study; following review by a central IRB, the local IRB accepts, modifies, or reviews its findings (an example is the National Cancer Institute's Central IRB process); and

- sites form a consortium and use the IRB of one of the sites to review a collaborative protocol.

The AAFP and the AAFP IRB plan to explore bringing experts and other stakeholders together in a small conference venue to discuss the models mentioned above, the strengths and weaknesses of these models for primary care settings, and the challenges these models present and how these challenges could be addressed. Some of these challenges include the reluctance of local IRBs to rely on the review of another IRB and new procedures that IRBs will need to create and implement to adopt a new review model.

Some of the questions that should be addressed at the conference regarding each model include:

- Will the local IRB have the ability to review the protocol for local context and resource requirements? Will the local IRB have the right not to allow a centrally approved protocol to be performed at their institution?

- What will be the relationship between the lead and local IRB, including adverse event reporting, auditing, and noncompliance?

- What will be the liability of a local IRB if it relies on the review of another IRB?

This conference will be important to represent at this conference the points of view of all groups that would be affected by the creation of a central form of review, including a variety of IRBs, academic institutions, health care systems, independent hospitals, professional organizations, funding agencies, and primary care researchers. Based on the outcome of this conference, the AAFP and the AAFP IRB may explore the possibility of implementing concrete steps toward adopting an improved IRB review model for practice-based research and family medicine. The AAFP NRN has also discussed the possibility of piloting a central review model in a single study.

In the meantime, the AAFP NRN and the AAFP IRB have discussed short-term solutions to the IRB challenge. The AAFP IRB agreed that members whose institutions have a Federalwide assurance
(FWA) with the OHRP and who are not required to report to a local IRB may request listing of the AAFP IRB on their FWA and development of an Authorization Agreement with the AAFP IRB. In this way, these members can fall under the jurisdiction of the AAFP IRB for our studies in which they participate and, consequently, would not have to complete and submit separate IRB applications to a local IRB or an independent IRB that may also be listed on the FWA.

The AAFP IRB also is considering going a step further in terms of accepting responsibility for PBRN studies and accepting the burden of extra reviews. Networks that have signed an affiliation agreement with the AAFP NRN may be allowed to submit protocols to the AAFP IRB. Consequently, if an affiliate network is not obliged to submit all protocols to a specific IRB for review, it may request listing of the AAFP IRB on their FWA in order to submit protocols to the AAFP IRB. This may benefit an affiliate network if the IRB to which it typically submits protocols is difficult to work with, does not understand the work of PBRNs, or is expensive.

\section{Conclusions}

IRB reviews and human subjects protections present challenges to PBRNs. The AAFP NRN has adopted several strategies to successfully address these challenges. However, the implementation of these strategies is a large drain on network resources, including staff time and the project timeline. The long-term solution for PBRN research in primary care may be the adoption of an alternative central IRB review model. The AAFP, the AAFP NRN, and the AAFP IRB have held preliminary discussions about next steps in working toward an alternative model and the place of the AAFP in a future model.

We thank the AAFP IRB for their understanding and dedication, in particular Tom Robinett, Jacqelyn Admire, and Dr. John Baumann. We also thank Elizabeth Staton, Dr. James Galliher, Dr. Wilson Pace, and Tom Stewart for assistance with this manuscript.

\section{References}

1. American Academy of Family Physicians. National Research Network (2006).

2. Burman WJ, Reves RR, Cohn DL, Schooley RT. Breaking the camel's back: multicenter clinical trials 
and local institutional review boards. Ann Intern Med 2001;134:152-7.

3. McWilliams R, Hoover-Fong J, Hamosh A, Beck S, Beaty T, Cutting G. Problematic variation in local institutional review of a multicenter genetic epidemiology study. JAMA 2003;290:360-6.

4. Gold JL, Dewa CS. Institutional review boards and multisite studies in health services research: is there a better way? Health Serv Res 2005;40:291-307.

5. Green LA, Lowery JC, Kowalski CP, Wyszewianski L. Impact of institutional review board practice variation on observational health services research. Health Serv Res 2006;41:214-30.

6. Wolf LE, Croughan M, Lo B. The challenges of IRB review and human subjects protections in practicebased research. Med Care 2002;40:521-9.

7. Wolf LE, Waldren JF, Lo B. Human subjects issues and IRB review in practice-based research. Ann Fam Med 2005;3 Suppl 11:S30-7.

8. Graham DG, Pace WD, Kappus JA, et al. Institutional review board approval of practice-based research network patient safety studies. Rockville (MD): Agency for Healthcare Research and Quality; 2005 Feb. Publication No. 050021 (3). p. 453-64.

9. Christoffel KK, Binns HJ, Stockman JA 3rd, et al. Practice-based research: opportunity and obstacles. Pediatrics 1988;82:399-406.
10. Dietrich AJ, O'Connor G, Keller A, et al. Will community physicians participate in rigorous studies of cancer control? The methodology and recruitment of a randomized trial of physician practices. Prog Clin Biol Res 1990;39:373-81.

11. Plane MB, Beasley JW, Wiesen P, McBride P, Underbakke G. Physician attitudes toward research study participation: a focus group. WMJ 1998;97: 49-51.

12. Alternative models of IRB review. Washington (DC): The Office for Human Research and Protections, National Institutes of Health, the Association of American Medical Colleges and the American Society of Clinical Oncology. Workshop Summary Report; 2005.

13. 45 CFR $\$ 46(2005)$.

14. 45 CFR 164.501, 164.508, 164.512 (i) (2003)

15. Office for Human Research Protections [homepage on the Internet]. Washington (DC): U.S. Department of Health \& Human Services. [updated 18 Oct 2006]. Available from: http://www.hhs.gov/ohrp/.

16. CITI [homepage on the Internet]. Available from: https://www.citiprogram.org/default.asp.

17. Future of Family Medicine Project Leadership Committee. The future of family medicine: a collaborative project of the family medicine community. Ann Fam Med. 2004;2:S3-32. 